\title{
Sleep quality, depression, and quality of life in elderly hemodialysis patients
}

This article was published in the following Dove Press journal: International Journal of Nephrology and Renovascular Disease 5 October 2012

Number of times this article has been viewed

\author{
Kultigin Turkmen' \\ Fatih Mehmet Erdurl \\ Ibrahim Guney ${ }^{2}$ \\ Abduzhappar Gaipov' \\ Faruk Turgut ${ }^{3}$ \\ Lutfullah Altintepe ${ }^{2}$ \\ Mustafa Saglam' \\ Halil Zeki Tonbul' \\ Emaad M Abdel-Rahman ${ }^{4}$ \\ 'Division of Nephrology, Meram \\ School of Medicine, Necmettin \\ Erbakan University, Meram, Konya, \\ Turkey; ${ }^{2}$ Division of Nephrology, \\ Meram Research and Training \\ Hospital, Meram, Konya, Turkey; \\ ${ }^{3}$ Division of Nephrology, Iskenderun \\ State Hospital, Iskenderun, Hatay, \\ Turkey; ${ }^{4}$ Division of Nephrology, \\ University of Virginia Health System, \\ Charlottesville, VA, USA
}

Correspondence: Kultigin Turkmen Department of Nephrology, Meram School of Medicine, Necmettin Erbakan University, Meram, Konya, 42090, Turkey Tel +90 5384927877 (mobile) Email mdkt20I0@yahoo.com
Objective: Both the incidence and the prevalence of end-stage renal disease (ESRD) in elderly patients are increasing worldwide. Elderly ESRD patients have been found to be more prone to depression than the general population. There are many studies that have addressed the relationship between sleep quality (SQ), depression, and health related quality of life (HRQoL) in ESRD patients, but previous studies have not confirmed the association in elderly hemodialysis (HD) patients. Therefore, the aim of the present study was to demonstrate this relationship in elderly HD patients.

Patients and methods: Sixty-three elderly HD patients ( 32 females and 31 males aged between 65 and 89 years) were included in this cross-sectional study. A modified Post-Sleep Inventory (PSI), the Medical Outcomes Study 36-item short form health survey, and the Beck Depression Inventory (BDI) were applied.

Results: The prevalence of poor sleepers (those with a PSI total sleep score [PSI-4 score] of 4 or higher) was $71 \%(45 / 63)$, and the prevalence of depression was $25 \%$ (16/63). Of the 45 poor sleepers, 15 had depression, defined as a BDI score of 17 or higher. Poor sleepers had a significantly higher rate of diabetes mellitus $(P=0.03)$, significantly higher total BDI scores, and lower Physical Component Scale scores (ie, lower HRQoL) than good sleepers. The PSI-4 score correlated negatively with Physical Component Scale $(r=-0.500, P<0.001)$ and Mental Component Scale scores $(r=-0.527, P<0.001)$ and it correlated positively with the BDI score $(r=0.606, P<0.001)$. In multivariate analysis, independent variables of PSI-4 score were BDI score (beta value $[\beta]=0.350, P<0.001)$, Mental Component Scale score $(\beta=-0.291$, $P<0.001)$, and age $(\beta=0.114, P=0.035)$.

Conclusion: Poor SQ is a very common issue and is associated with both depression and lower HRQoL in elderly HD patients.

Keywords: health-related quality of life, end-stage renal disease, poor sleep quality, Post-Sleep Inventory, Beck Depression Inventory

\section{Introduction}

The prevalence of depression in elderly patients (those aged 65 years or older) may be as high as $40 \%$ in hospital and nursing home settings and $8 \%-15 \%$ in community settings. ${ }^{1}$ Depression is a major contributor to health care costs associated with older populations, and it is projected to be the leading cause of disease burden in older populations by the year 2020., ${ }^{2,3}$ Both the incidence and the prevalence of end-stage renal disease (ESRD) in elderly patients are also increasing worldwide. ${ }^{4,5}$ Elderly ESRD patients, especially those with vascular risk factors, have been found to be more prone to depression than the general population. ${ }^{6}$ In a meta-analysis, both the presence of chronic disease and poor self-reported health status were found to be risk 
factors for increased depression among the elderly, with poor self-reported health appearing to be more closely associated with depression than the presence of chronic disease. ${ }^{7}$ The prognosis of these depressive states is poor. Clinical depression is a common psychiatric disorder and is associated with increased morbidity and mortality in ESRD patients. ${ }^{8}$

Poor sleep quality (SQ) is not uncommon in hemodialysis (HD) patients; the prevalence of poor SQ in this population has been shown to range from $41 \%$ to $83 \%$. $^{9,10}$ Previous studies have shown that poor SQ in HD patients is associated with female sex, advanced age, depression, cardiovascular disease, dialysis vintage, poor dialysis quality, and poor health-related quality of life (HRQoL). ${ }^{11,12,13}$ It is widely accepted that HRQoL is an important outcome of health care, both in the general population and in ESRD patients. ${ }^{14}$ Also, as stated earlier, depressive mood has been found to be associated with morbidity and mortality in ESRD patients. ${ }^{15,16}$ There are many studies that have addressed the relationship between SQ, depression, and HRQoL in the general population; ${ }^{17-20}$ however, the data about SQ, depression, and HRQoL in elderly HD patients are limited. Kutner et $\mathrm{al}^{21}$ reported that older dialysis patients are significantly prone to restless sleep. Because SQ is a modifiable risk factor and there are implications that improving SQ can also cure depression and improve HRQoL, the exact relationship between these issues should be examined in elderly HD patients. Therefore, the aim of this study was to investigate the association between SQ, depression, HRQoL, and independent variables of SQ in elderly HD patients.

\section{Patients and methods}

The present study is a subgroup analysis of a previous study by the authors. ${ }^{11}$ A total of 233 ESRD patients receiving HD three times weekly over a period of more than 3 months at five centers in Konya, Turkey, between February and June 2006 were evaluated in this cross-sectional study. Patients aged 65 years or older were accepted as "elderly" patients. A review of medical records, including information on age, sex, weight, height, duration of renal replacement therapy, medications, and primary cause of ESRD, was undertaken. Exclusion criteria included (1) severe cognitive impairment, (2) inability to answer the questionnaire, (3) treatment with a sleep medication, (4) presence of chronic pain, (5) presence of chronic obstructive pulmonary disease, (6) presence of psychosis, (7) a past history of sleep disorders including dyssomnias (frequent awakening, hypervigilance, etc) and parasomnias (sleepwalking, bruxism, etc), (8) a past history of sleep apnea syndrome, and (9) patients younger than 65 years of age. Of the 233 ESRD patients evaluated, 63 elderly patients (aged between 65 and 89 years) who were willing to participate and who met the inclusion criteria were included in the study.

HD patients received dialysis three times a week for 4 hours each time. Patients were dialyzed with a standard bicarbonate-containing dialysate bath, using a biocompatible HD membrane (Fresenius Polysulfone ${ }^{\circledR}$, FX-80 series; Fresenius Medical Care, Bad Homburg, Germany). Dialysate flow rates were set at $500 \mathrm{~mL}$ per minute and blood flow rates were kept between 250 and $300 \mathrm{~mL}$ per minute. Both the systolic and the diastolic blood pressures of patients were measured in the upright sitting position after 5 minutes or more of rest, using an Erka sphygmomanometer [PMS (Instruments) Ltd, Berkshire, UK] with an appropriate cuff size. Two readings were recorded for each individual, and the mean value of these two readings was defined as the blood pressure. Patients with a systolic blood pressure greater than $140 \mathrm{mmHg}$ and a diastolic blood pressure of $90 \mathrm{mmHg}$ or those who were already on antihypertensive treatment were assumed to be hypertensive.

In patients receiving $\mathrm{HD}$, venous blood samples for biochemical analyses were drawn after an overnight fast before the midweek HD session. All biochemical analyses including glucose, creatinine, total cholesterol, low-density lipoprotein cholesterol, high-density lipoprotein cholesterol, and plasma triglyceride concentrations - were performed using an oxidase-based technique and were measured by a Roche/Hitachi Modular System (Roche Diagnostics GmbH, Mannheim, Germany) in the Central Biochemistry Laboratory of Selcuk University, Konya, Turkey.

The institutional medical ethics committee of Selçuk University, Konya, Turkey, approved the study protocol, and written informed consent was obtained from all subjects included in the study.

\section{Evaluation of SQ}

To evaluate SQ, a modified Post-Sleep Inventory (PSI) was applied to all patients. The PSI was developed by Webb et $\mathrm{al}^{22}$ to permit an adequate description of subjective responses to a preceding period of sleep. The PSI involves a questionnaire with three groups of opposing statements separated by an analog rating scale from 0 to 1 . A score of 0 reflects a positive opinion about the patient's SQ, while a score of 1 reflects a negative opinion. The aim is to classify the patient's understanding about his or her SQ in terms of feelings at bedtime (PSI-1 score), quality of nocturnal sleep (PSI-2 score), and feelings at awakening (PSI-3 score). For 
this study, a total sleep score (PSI-4 score) was calculated by adding the PSI-1, PSI-2, and PSI-3 scores for each patient. The PSI-4 score could indicate no sleep problems at all (0), or mild (1-3), moderate (4-6), severe (7-9), or very severe (10-12) sleep problems.

\section{Evaluation of HRQoL}

The Medical Outcomes Study 36-item short form health survey was used to evaluate HRQoL. ${ }^{23}$ The test comprises 36 items, which are assigned to eight dimensions - namely, (1) physical functioning (ten items), (2) physical role functioning (four items), (3) bodily pain (two items), (4) general health status (five items), (5) vitality (four items), (6) social functioning (two items), (7) emotional role functioning (three items), and (8) mental health (five items). Each scale is scored within a range of $0-100-$ the higher the score, the better the HRQoL indicated. While the first four items constitute the Physical Component Scale (PCS), the remaining four items constitute the Mental Component Scale (MCS). These two summary scales have been shown to adequately represent values of their individual scale components with $80 \%$ and $85 \%$ variability, respectively. ${ }^{24}$ This scale has been validated and is commonly used in patients with ESRD. ${ }^{25,26}$

\section{Evaluation of depression}

Depression was assessed using the Beck Depression Inventory (BDI), which had been validated and is commonly used in patients with ESRD. ${ }^{15,16}$ It has been reported that $85 \%$ of Western dialysis patients with BDI scores of 11 or higher meet the Diagnostic and Statistical Manual of Mental Disorders, fourth edition, criteria for diagnosis of major depression. ${ }^{15}$ In Hisli's ${ }^{27}$ validation and reliability study in a Turkish population, a BDI score of 17 or higher was determined as the cutoff value for the diagnosis of depression. Depression was also defined as a BDI score of 17 or higher in the present study. ${ }^{28}$

\section{Statistical analysis}

All calculations were performed using a standard statistical software package (SPSS v 13.0 for Windows; SPSS Inc, Chicago, IL). The data are presented as the mean plus or minus the standard deviation, unless otherwise specified. The proportion of subjects with a PSI- 4 score of 4 or higher determined the prevalence of poor sleepers. Student's $t$-test was used to compare the means of normally distributed variables and the Mann-Whitney U test was used for variables that were not normally distributed. Differences among categorical variables were analyzed using the chi-square test or the two-tailed Fisher's exact test as appropriate. Spearman correlation coefficients were used to examine associations between continuous variables. Multiple linear regression analysis with forward stepwise selection $(P=0.05)$ was performed to identify factors independently associated with the PSI-4 score. The level of significance ( $P$-value) was 0.05 for all comparisons.

\section{Results}

The sociodemographic and clinical features of the patients in this study are shown in Table 1 . The mean age of the patients was $70.5 \pm 4.7$ years and the mean dialysis vintage was $48.3 \pm 35$ months. The patients had a range of conditions: diabetic nephropathy $(\mathrm{n}=19)$, chronic glomerulonephritis $(\mathrm{n}=2)$, hypertensive nephropathy $(\mathrm{n}=15)$, polycystic kidney disease $(n=2)$, chronic pyelonephritis $(n=5)$, and unknown $(n=20)$.

The mean and median PSI-4 scores were $6.4 \pm 3.6$ and 6.0 (range, 0-12), respectively. The prevalence of poor sleepers (defined as those having a PSI-4 score of 4 or higher) was $71 \%(45 / 63)$, and the prevalence of depression in elderly HD patients was $25 \%(16 / 63)$ in this study.

The sociodemographic characteristics of the good sleepers compared with the poor sleepers are shown in Table 2. Of the 45 poor sleepers in this study, 15 had depression (ie, a BDI score of 17 or higher); however, of the 18 good

Table I Sociodemographic and clinical characteristics of elderly hemodialysis patients $(n=63)$

\begin{tabular}{ll}
\hline Parameter & Statistic \\
\hline Age (years)* & $70.5 \pm 4.7$ \\
Gender (M/F) & $31 / 32$ \\
Presence of DM [n (\%)] & $19(30 \%)$ \\
Presence of hypertension [n (\%)] & $21(33 \%)$ \\
Smoking [n (\%)] & $5(8 \%)$ \\
Employed [n (\%)] & $9(14 \%)$ \\
Dialysis vintage (months)* & $48.3 \pm 35$ \\
BMI (kg/m²) & $23.9 \pm 3.8$ \\
SBP (mmHg)* & $129 \pm 20$ \\
DBP (mmHg)* & $74 \pm 10$ \\
Serum albumin (g/dL)* & $4.0 \pm 0.4$ \\
Hemoglobin (g/dL)* & $10.9 \pm 1.2$ \\
Total cholesterol (mg/dL)* & $182 \pm 51$ \\
Triglycerides (mg/dL)* & $172 \pm 113$ \\
Calcium (mg/dL)* & $8.9 \pm 0.7$ \\
Phosphorus (mg/dL)* & $4.5 \pm 1.2$ \\
PTH (pg/mL)* & $254 \pm 240$ \\
Kt/V* & $1.29 \pm 0.2$ \\
\hline Not &
\end{tabular}

Note: *Data presented as mean plus or minus standard deviation.

Abbreviations: $\mathrm{BMI}$, body mass index; DBP, diastolic blood pressure; DM, diabetes mellitus; F, female; M, male; PTH, parathormon; SBP, systolic blood pressure. 
Table 2 Sociodemographic and clinical characteristics of good versus poor sleepers

\begin{tabular}{|c|c|c|c|}
\hline Parameter & $\begin{array}{l}\text { Good sleepers } \\
(\text { PSI-4 score }<4) \\
(n=\mid 8)\end{array}$ & $\begin{array}{l}\text { Poor sleepers } \\
(P S I-4 \text { score } \geq 4) \\
(n=45)\end{array}$ & $P$-value \\
\hline Age (years)* & $70.4 \pm 5.0$ & $70.6 \pm 4.6$ & 0.87 \\
\hline Female [n (\%)] & $8(44 \%)$ & $24(53 \%)$ & 0.58 \\
\hline Presence of DM [n (\%)] & $2(11 \%)$ & 17 (38\%) & 0.03 \\
\hline Presence of hypertension [n (\%)] & $9(50 \%)$ & $12(27)$ & 0.69 \\
\hline Presence of depression & I (6\%) & $15(33 \%)$ & 0.02 \\
\hline \multicolumn{4}{|l|}{$(B D I$ score $\geq 17)[n(\%)]$} \\
\hline Smoker [n (\%)] & $2(11 \%)$ & $3(7 \%)$ & 0.49 \\
\hline Employed [n (\%)] & $2(11 \%)$ & $7(16 \%)$ & 0.49 \\
\hline Dialysis vintage (months)* & $59.7 \pm 45.8$ & $43.8 \pm 29.1$ & 0.18 \\
\hline BMI $\left(\mathrm{kg} / \mathrm{m}^{2}\right)^{*}$ & $23.7 \pm 3.7$ & $24.0 \pm 3.7$ & 0.81 \\
\hline $\mathrm{SBP}(\mathrm{mmHg})^{*}$ & $|32 \pm 2|$ & $127 \pm 19$ & 0.44 \\
\hline $\mathrm{DBP}(\mathrm{mmHg})^{*}$ & $76 \pm 11$ & $73 \pm 10$ & 0.41 \\
\hline Serum albumin $(g / d L)^{*}$ & $4.0 \pm 0.4$ & $4.1 \pm 0.4$ & 0.69 \\
\hline Hemoglobin $(g / d L) *$ & $10.1 \pm 1.2$ & $10.8 \pm 1.1$ & 0.32 \\
\hline Total cholesterol (mg/dL)* & $17 \mid \pm 43$ & $187 \pm 54$ & 0.28 \\
\hline Triglycerides $(\mathrm{mg} / \mathrm{dL})^{*}$ & $185 \pm 186$ & $167 \pm 64$ & 0.36 \\
\hline Calcium (mg/dL)* & $8.8 \pm 0.7$ & $9.0 \pm 0.7$ & 0.35 \\
\hline Phosphorus (mg/dL)* & $4.1 \pm 1.2$ & $4.7 \pm 1.1$ & 0.05 \\
\hline $\mathrm{Kt} / \mathrm{V}^{*}$ & $1.33 \pm 0.2$ & $1.27 \pm 0.2$ & 0.32 \\
\hline Total BDI score* & $10.4 \pm 5.3$ & $15.1 \pm 8.9$ & 0.03 \\
\hline PCS score* & $56.9 \pm 21.8$ & $43.5 \pm 22$ & 0.04 \\
\hline MCS score* & $66.7 \pm 20.5$ & $56 \pm 23$ & 0.06 \\
\hline
\end{tabular}

Note: *Data presented as mean plus or minus standard deviation.

Abbreviations: BDI, Beck Depression Inventory; BMI, body mass index; DBP, diastolic blood pressure; DM, diabetes mellitus; MCS, Mental Component Scale; PCS, Physical Component Scale; PSI-4 score, Post-Sleep Inventory total sleep score; SBP, systolic blood pressure.

sleepers in this study, only one had depression. The presence of diabetes was significantly higher in poor sleepers than in good sleepers ( $38 \%$ and $11 \%$, respectively; $P=0.03)$. The mean BDI, PCS, and MCS scores of good sleepers were $10.4 \pm 5.3,56.9 \pm 21.8$, and $66.7 \pm 20.5$, respectively. Poor sleepers had significantly higher total BDI scores and lower PCS scores than good sleepers (Table 2). A comparison of the mean MCS scores of poor sleepers and good sleepers shows $56 \pm 23$ and $66.7 \pm 20.5$, respectively $(P=0.06)$.

The correlations between the PSI-4 score and other continuous variables were studied. There was a significant negative correlation between PSI-4 score and PCS score $(r=-0.500$, $P<0.001$ ) (Figure 1) and between PSI-4 score and MCS score $(r=-0.527, P<0.001)$ (Figure 2). Additionally, there was a positive correlation of PSI-4 score with BDI score ( $r=0.606, P<0.001$ ) (Figure 3 ). In multivariate analysis, the independent variables of PSI-4 score were BDI score (beta value $[\beta]=0.350, P<0.001), \operatorname{MCS}$ score $(\beta=-0.291$, $P<0.001)$, and age $(\beta=0.114, P=0.035)$ (Table 3$)$. All of the dimensions of HRQoL were significantly higher in good sleepers than in poor sleepers (Table 4).

The authors also analyzed whether any correlation could be found between BDI score and smoking status, hypertension, and biochemical parameters (including parathormon, hemoglobin, and albumin levels). No relationship was found between PSI-4 score and smoking status, hypertension, and biochemical parameters. Also,

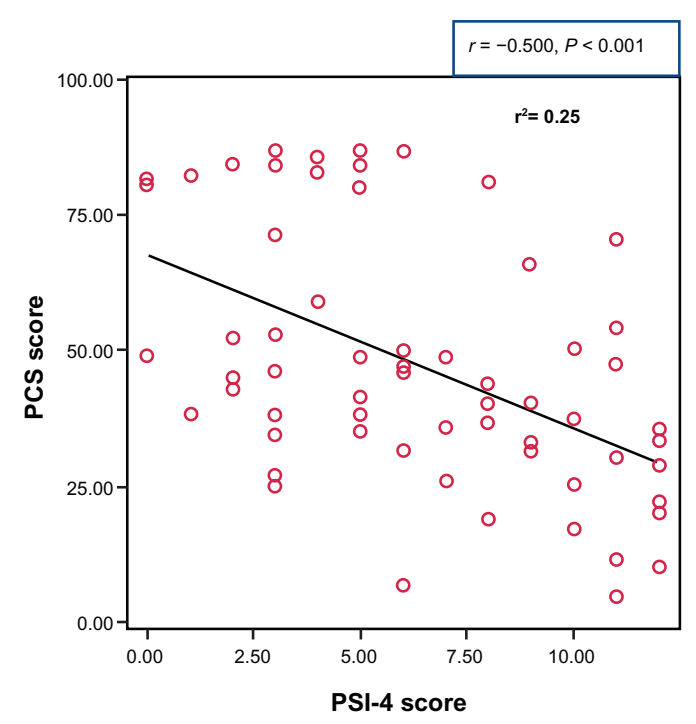

Figure I The correlation between Physical Component Scale (PCS) score and PostSleep Inventory total sleep score (PSI-4 score). 


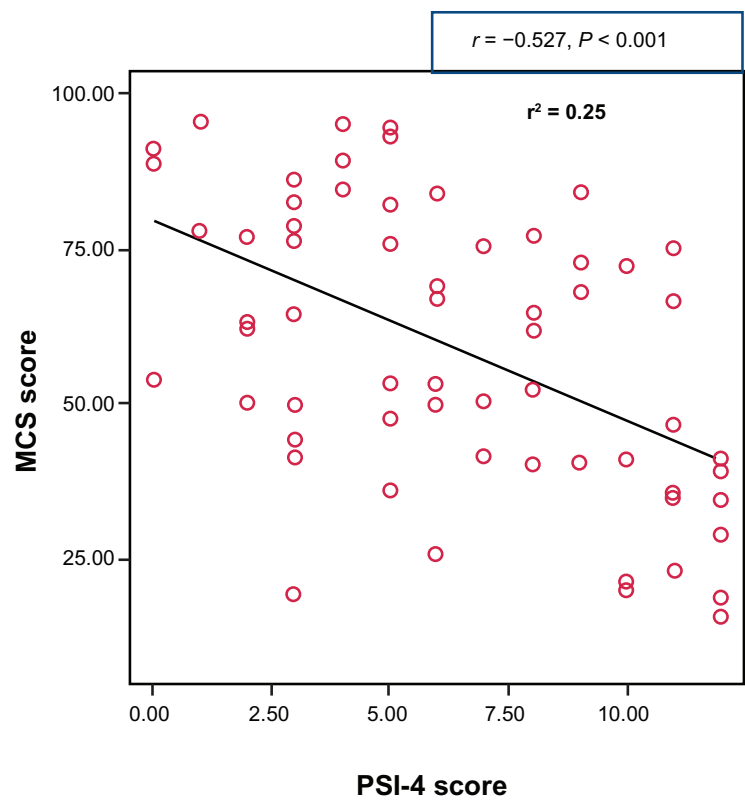

Figure 2 The correlation between Mental Component Scale (MCS) score and PostSleep Inventory total sleep score (PSI-4 score).

none of the dimensions of HRQoL were correlated with these parameters (data not shown).

\section{Discussion}

There were four main findings of this study. First, the authors found the prevalence of poor sleepers (those with a PSI-4 score of 4 or higher) to be $71 \%$ in the elderly HD patients. Second, poor sleepers had significantly higher

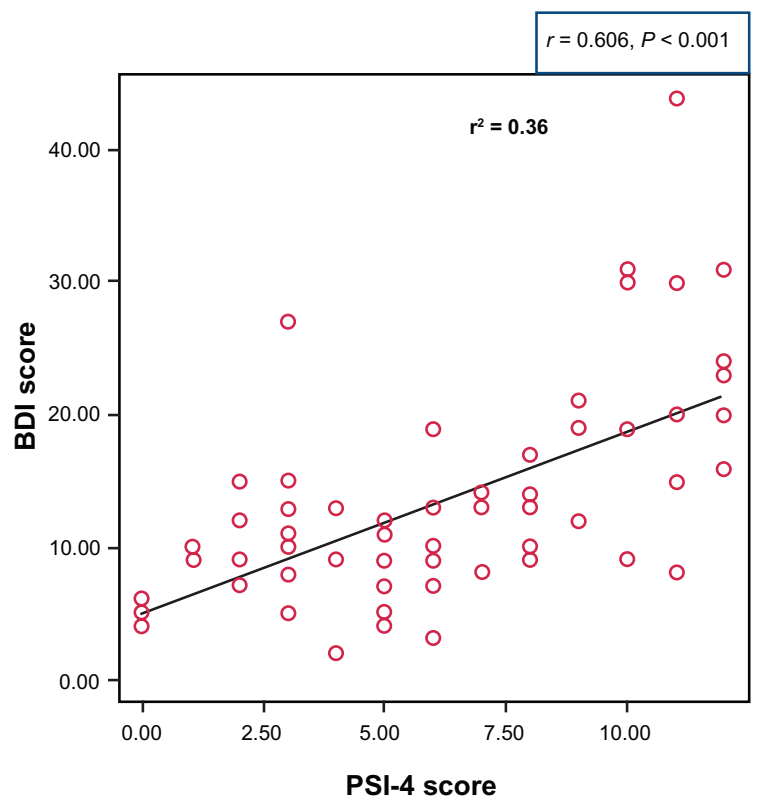

Figure 3 The correlation between Beck Depression Inventory (BDI) score and Post-Sleep Inventory total sleep score (PSI-4 score).
Table 3 Multiple linear regression models of independent variables of Post-Sleep Inventory total sleep score

\begin{tabular}{llll}
\hline Variable & Beta value & $\boldsymbol{P}$-value & $\mathbf{9 5 \%} \mathbf{C l}$ \\
\hline BDI score & 0.350 & $<0.001$ & $0.086-0.193$ \\
MCS score & -0.291 & $<0.001$ & -0.068 to -0.025 \\
Age & 0.114 & 0.035 & $0.002-0.005 \mathrm{I}$
\end{tabular}

Abbreviations: BDI, Beck Depression Inventory; $\mathrm{Cl}$, confidence interval; $\mathrm{MCS}$, Mental Component Scale.

total BDI scores and lower PCS scores than good sleepers. Third, there was a significant inverse relationship between the PSI-4 score and the PCS and MCS scores; however, a positive correlation was found between the PSI-4 score and the BDI score. Fourth, in the multivariate analysis, the independent variables of the PSI-4 score were found to be BDI score, MCS score, and age.

To the best of the authors' knowledge, this is the first study evaluating the relationship between SQ, depression, and HRQoL in elderly HD patients. In previous studies, the prevalence of poor SQ in HD patients has been reported as $41 \%-83 \% .{ }^{9-11}$ In the present study, the prevalence of poor SQ in elderly HD patients was found to be $71 \%$ (45/63). This result shows that the prevalence of poor SQ in elderly HD patients is high, as also seen in the general HD population.

In the present study, the prevalence of depression in elderly HD patients was found to be $25 \%$ (16/63), which is similar to that found in previous studies. ${ }^{28,29}$ Several previous studies have reported a remarkable relationship between sleep alterations and depression in the general population. Elder et $\mathrm{al}^{9}$ showed that depression was the only predictor of poor SQ. Along the same lines, Pai et $\mathrm{al}^{10}$ also reported that poor sleepers had a higher BDI score than good sleepers and that this factor was one of the predictors of poor SQ in patients. Furthermore, Güney et $\mathrm{a}^{30}$ concluded that poor SQ is a significant problem in peritoneal dialysis patients and suggested that regular assessment and management of SQ may be important for improvement of depression therapy in this population. Recently, Araujo et $\mathrm{al}^{31}$ demonstrated that depressive symptoms are also independently associated with poor SQ in 400 patients on chronic HD. However, this relationship has been demonstrated less in elderly HD patients. In the present study, the authors observed that the elderly poor sleepers had a higher BDI score than the elderly good sleepers, which indicates that poor SQ is also associated with depression in the elderly HD population.

ESRD affects SQ at least by increasing the incidence of some specific sleep diseases. ${ }^{32-34}$ Previous studies have 
Table 4 Dimensions of health-related quality of life (HRQoL) among good and poor sleepers

\begin{tabular}{llll}
\hline Dimension of HRQoL & Good sleepers (PSI-4 score $<4) *$ & Poor sleepers (PSI-4 score $\geq 4) *$ & P-value \\
\hline Mental health & $73.5 \pm 15.6$ & $61.7 \pm 21.2$ & $<0.001$ \\
General health status & $56.5 \pm 23.2$ & $41.8 \pm 23.2$ & $<0.00 I$ \\
Vitality & $69.5 \pm 19.4$ & $54.7 \pm 21.6$ & $<0.00 \mathrm{I}$ \\
Social functioning & $90.5 \pm 17.8$ & $76.4 \pm 28.4$ & $<0.00 \mathrm{I}$ \\
Physical role functioning & $58.2 \pm 46.4$ & $42.9 \pm 47.4$ & 0.015 \\
Emotional role functioning & $63.7 \pm 42$ & $45.5 \pm 44$ & 0.002 \\
Bodily pain & $77.9 \pm 26.5$ & $69.6 \pm 28.5$ & 0.024 \\
Physical functioning & $64.2 \pm 30.2$ & $53.1 \pm 32.7$ & 0.01 \\
\hline
\end{tabular}

Note: *Data presented as mean plus or minus standard deviation.

Abbreviation: PSI-4 score, Post-Sleep Inventory total sleep score.

found SQ to be associated with HRQoL in ESRD patients, and patients with good SQ have been found more likely to have high MCS and PCS scores (ie, good HRQoL). ${ }^{9,11,35}$ Recently, Li et al ${ }^{36}$ demonstrated that SQ is also affected by malnutrition and calcium-phosphorus product in continuous ambulatory peritoneal dialysis patients. In the present study, the poor sleepers had lower PCS scores (ie, lower HRQoL) than the good sleepers, and there was a significant negative correlation between PSI-4 score and both MCS and PCS scores. In multiple linear regression analysis, BDI score, MCS score, and age were found to be independent variables of the PSI-4 score. These results were consistent with results from the authors' previous study evaluating HD patients. ${ }^{11}$

There are conflicting results in the literature regarding the role of gender, smoking status, and comorbidity in sleep diseases in patients with ESRD. Some studies have reported that female sex has a negative impact on $S Q,{ }^{9,10,12}$ while other studies have reported that SQ is not affected by gender. ${ }^{30,35,37-39}$ Smoking cigarettes was also found to be associated with sleep diseases in some ${ }^{9,38}$ but not all studies. ${ }^{12,37}$ In the present study, only $8 \%$ of the patients were smokers and there was no relationship found between smoking and sleep diseases. This result could be attributed to the small sample size of elderly HD patients who smoked cigarettes, compared with the number of elderly HD patients who did not smoke.

Restless legs syndrome (RLS) has been found to be associated with diabetes in the general population..$^{40,41}$ Although some of the increased risk of RLS in diabetes appears to be mediated through the presence of peripheral neuropathy, the association between RLS and diabetes remains significant, even in patients without neuropathy. ${ }^{42}$ Epidemiologic data are increasingly attesting to the negative impact of RLS upon health. RLS is significantly associated with diminished quality of life ${ }^{43}$ and depression. ${ }^{44}$ RLS was not explored in the patients in the present study; however, the rate of diabetes was higher in poor sleepers than in good sleepers (38\% and $11 \%$, respectively) - this might explain the poor quality of sleep noted in the patients in the present study.

Cognitive behavioral therapy (CBT) is a psychotherapeutic approach that addresses dysfunctional emotions, behaviors, and cognitions through a goal-oriented, systematic process. CBT has six phases: (1) assessment, (2) reconceptualization, (3) skills acquisition, (4) skills consolidation and application training, (5) generalization and maintenance, and (6) post-treatment assessment follow-up. ${ }^{45} \mathrm{CBT}$ has been shown to have a role in the treatment plans for anxiety disorders ${ }^{46}$ and depression. ${ }^{47} \mathrm{CBT}$ has been found to be an effective treatment for sleep disturbance and has been found to reduce inflammation in HD patients. ${ }^{48}$ Recently, Chen et al ${ }^{48}$ demonstrated that CBT is effective for correcting disorganized sleep patterns and that it reduced inflammation and oxidative stress in 72 sleep - disturbed HD patients. Therefore, CBT should be encouraged in HD patients.

This study had three main limitations. First, this was a cross-sectional analysis; this was not a prospective controlled study, so cause-and-effect relationships cannot be drawn from the findings. Second, the sample size was relatively small. Third, the diagnosis of sleep apnea syndrome depended on each patient's past history; therefore, the authors could not ensure the diagnosis of sleep apnea syndrome was correct for each patient.

\section{Conclusion}

In conclusion, poor SQ is a very common issue and is found to be associated with depression and poor HRQoL in elderly HD patients. Social and economic environment, family status, life events, personality, and biological consequences of aging may be additional determinants of depression, poor SQ, and poor HRQoL in this population. Assessment of SQ and bystander factors such as depression and HRQoL and 
treatment of these factors should become a part of treatment for elderly dialysis patients.

\section{Disclosure}

The authors report no conflicts of interest in this work.

\section{References}

1. Leon FG, Ashton AK, D'Mello DA, et al. Depression and comorbid medical illness: therapeutic and diagnostic challenges. J Fam Pract. 2003;Suppl:S19-S33.

2. Goodwin RD. Association between physical activity and mental disorders among adults in the United States. Prev Med. 2003;36(6):698-703.

3. Katon WJ, Lin E, Russo J, Unutzer J. Increased medical costs of a population-based sample of depressed elderly patients. Arch Gen Psychiatry. 2003;60(9):897-903.

4. Kurella M, Covinsky KE, Collins AJ, Chertow GM. Octogenarians and nonagenarians starting dialysis in the United States. Ann Intern Med. 2007;146(3):177-183.

5. Collins AJ, Foley R, Herzog C, et al. Excerpts from the United States Renal Data System 2007 annual data report. Am J Kidney Dis. 2008; 51(1 Suppl 1):S1-S320.

6. Prince MJ, Beekman AT, Deeg DJ, et al. Depression symptoms in late life assessed using the EURO-D scale: effect of age, gender and marital status in 14 European centres. Br J Psychiatry. 1999;174:339-345.

7. Chang-Quan H, Xue-Mei Z, Bi-Rong D, Zhen-Chan L, Ji-Rong Y, Qing-Xiu L. Health status and risk for depression among the elderly: a meta-analysis of published literature. Age Ageing. 2010;39(1): 23-30.

8. Lopes AA, Bragg J, Young E, et al; for Dialysis Outcomes and Practice Patterns Study. Depression as a predictor of mortality and hospitalization among hemodialysis patients in the United States and Europe Kidney Int. 2002;62(1):199-207.

9. Elder SJ, Pisoni RL, Akizawa T, et al. Sleep quality predicts quality of life and mortality risk in haemodialysis patients: results from the Dialysis Outcomes and Practice Patterns Study (DOPPS). Nephrol Dial Transplant. 2008;23(3):998-1004.

10. Pai MF, Hsu SP, Yang SY, Ho TI, Lai CF, Peng YS. Sleep disturbance in chronic hemodialysis patients: the impact of depression and anemia. Ren Fail. 2007;29(6):673-677.

11. Güney I, Atalay H, Solak Y, et al. Predictors of sleep quality in hemodialysis patients. Int J Artif Organs. 2010;33(3):154-160.

12. Sabbatini M, Minale B, Crispo A, et al. Insomnia in maintenance haemodialysis patients. Nephrol Dial Transplant. 2002;17(5):852-856.

13. Turkmen K, Yazici R, Solak Y, et al. Health-related quality of life, sleep quality, and depression in peritoneal dialysis and hemodialysis patients. Hemodial Int. 2012;16(2):198-206.

14. Wu AW, Fink NE, Marsh-Manzi JV, et al. Changes in quality of life during hemodialysis and peritoneal dialysis treatment: generic and disease specific measures. J Am Soc Nephrol. 2004;15(3): 743-753.

15. Kimmel PL, Peterson RA, Weihs KL, et al. Multiple measurements of depression predict mortality in a longitudinal study of chronic hemodialysis outpatients. Kidney Int. 2000;57(5):2093-2098.

16. Watnick S, Kirwin P, Mahnensmith R, Concato J. The prevalence and treatment of depression among patients starting dialysis. Am J Kidney Dis. 2003;41(1):105-110.

17. Motivala SJ, Levin MJ, Oxman MN, Irwin MR. Impairments in health functioning and sleep quality in older adults with a history of depression. J Am Geriatr Soc. 2006;54(8):1184-1191.

18. Morgan K, Clarke D. Longitudinal trends in late-life insomnia: implications for prescribing. Age Ageing. 1997;26(3):179-184.

19. Habte-Gabr E, Wallace RB, Colsher PL, Hulbert JR, White LR, Smith IM. Sleep patterns in rural elders: demographic, health, and psychobehavioral correlates. J Clin Epidemiol. 1991;44(1):5-13.
20. Schechtman KB, Kutner NG, Wallace RB, Buchner DM, Ory MG Gender, self-reported depressive symptoms, and sleep disturbance among older community-dwelling persons. FICSIT group. Frailty and Injuries: Cooperative Studies of Intervention Techniques. J Psychosom Res. 1997;43(5):513-527.

21. Kutner NG, Bliwise DL, Brogan D, Zhang R. Race and restless sleep complaint in older chronic dialysis patients and nondialysis community controls. J Gerontol B Psychol Sci Soc Sci. 2001;56(3): P170-P175.

22. Webb WB, Bonnet MH, White RM. State and trait correlates of sleep stages. Psychol Rep. 1976;38(3 Pt 2):1181-1182.

23. Ware JE Jr, Sherbourne CD. The MOS 36-item short-form health survey (SF-36): I. Conceptual framework and item selection. Med Care. 1992;30(6):473-483.

24. Ware JE Jr, Kosinski M, Bayliss MS, McHorney CA, Rogers WH, Raczek A. Comparison of methods for the scoring and statistical analysis of SF-36 health profile and summary measures: summary of results from the Medical Outcomes Study. Med Care. 1995; 33(Suppl 4):AS264-AS279

25. Johansen KL, Painter P, Kent-Braun JA, et al. Validation of questionnaires to estimate physical activity and functioning in end-stage renal disease. Kidney Int. 2001;59(3):1121-1127.

26. Kušleikaite N, Bumblytė IA, Kuzminskis V, Vaičiūnienè R. The association between health-related quality of life and mortality among hemodialysis patients. Medicina (Kaunas). 2010;46(8):531-537.

27. Hisli N. A study about the validation of Beck Depression Inventory. Beck Depresyon Envanterinin geçerliliği üzerine bir çalışma. Psikoloji Dergisi. 1998;6:118-122. Turkish

28. Balogun RA, Turgut F, Balogun SA, Holroyd S, Abdel-Rahman EM Screening for depression in elderly hemodialysis patients. Nephron Clin Pract. 2011;118(2):c72-c77.

29. Brown EA, Johansson L, Farrington K, et al. Broadening options for long-term dialysis in the elderly (BOLDE): differences in quality of life on peritoneal dialysis compared to haemodialysis for older patients. Nephrol Dial Transplant. 2010;25(11):3755-3763.

30. Güney I, Biyik M, Yeksan M, et al. Sleep quality and depression in peritoneal dialysis patients. Ren Fail. 2008;30(10):1017-1022.

31. Araujo SM, de Bruin VM, Daher Ede D, Almeida GH, Medeiros CA, de Bruin PF. Risk factors for depressive symptoms in a large population on chronic hemodialysis. Int Urol Nephrol. 2012;44(4):1229-1235.

32. Hanly PJ, Pierratos A. Improvement of sleep apnea in patients with chronic renal failure who undergo nocturnal hemodialysis. $N$ Engl $J$ Med. 2001;344(2):102-107.

33. Cengić B, Resić H, Spasovski G, Avdić E, Alajbegović A. Quality of sleep in patients undergoing hemodialysis. Int Urol Nephrol. 2012; 44(2):557-567.

34. Molnar MZ, Novak M, Mucsi I. Sleep disorders and quality of life in renal transplant recipients. Int Urol Nephrol. 2009;41(2):373-382.

35. Unruh ML, Buysse DJ, Dew MA, et al; for Choices for Healthy Outcomes in Caring for End-Stage Renal Disease (CHOICE) Study. Sleep quality and its correlates in the first year of dialysis. Clin J Am Soc Nephrol. 2006;1(4):802-810.

36. Li J, Guo Q, Ye X, et al. Prevalence and risk factors of sleep disturbance in continuous ambulatory peritoneal dialysis patients in Guangzhou, southern China. Int Urol Nephrol. 2012;44(3):929-936.

37. De Vecchi A, Finazzi S, Padalino R, et al. Sleep disorders in peritoneal and haemodialysis patients as assessed by a self-administered questionnaire. Int J Artif Organs. 2000;23(4):237-242.

38. Merlino G, Piani A, Dolso P, et al. Sleep disorders in patients with endstage renal disease undergoing dialysis therapy. Nephrol Dial Transplant. 2006;21(1):184-190.

39. Chen WC, Lim PS, Wu WC, et al. Sleep behavior disorders in a large cohort of Chinese (Taiwanese) patients maintained by long-term hemodialysis. Am J Kidney Dis. 2006;48(2):277-284.

40. Phillips B, Young T, Finn L, Asher K, Hening WA, Purvis C. Epidemiology of restless legs symptoms in adults. Arch Intern Med. 2000;160(14):2137-2141 
41. Berger K, Luedemann J, Trenkwalder C, John U, Kessler C. Sex and the risk of restless legs syndrome in the general population. Arch Intern Med. 2004;164(2):196-202.

42. Benediktsdottir B, Janson C, Lindberg E, et al. Prevalence of restless legs syndrome among adults in Iceland and Sweden: lung function, comorbidity, ferritin, biomarkers and quality of life. Sleep Med. 2010; 11(10):1043-1048.

43. Ulfberg J, Nyström B, Carter N, Edling C. Restless legs syndrome among working-aged women. Eur Neurol. 2001;46(1):17-19.

44. Allen RP, Walters AS, Montplaisir J, et al. Restless legs syndrome prevalence and impact: REST general population study. Arch Intern Med. 2005;165(11):1286-1292.
45. Gatchel RJ, Rollings KH. Evidence-informed management of chronic low back pain with cognitive behavioral therapy. Spine J. 2008;8(1):40-44.

46. Otte C. Cognitive behavioral therapy in anxiety disorders: current state of the evidence. Dialogues Clin Neurosci. 2011;13(4):413-421.

47. Driessen E, Hollon SD. Cognitive behavioral therapy for mood disorders: efficacy, moderators and mediators. Psychiatr Clin North Am. 2010;33(3):537-555.

48. Chen HY, Cheng IC, Pan YJ, et al. Cognitive-behavioral therapy for sleep disturbance decreases inflammatory cytokines and oxidative stress in hemodialysis patients. Kidney Int. 2011;80(4):415-422.

\section{Publish your work in this journal}

The International Journal of Nephrology and Renovascular Disease is an international, peer-reviewed open-access journal focusing on the pathophysiology of the kidney and vascular supply. Epidemiology, screening, diagnosis, and treatment interventions are covered as well as basic science, biochemical and immunological studies. The journal welcomes original research, clinical studies, reviews \& evaluations, expert opinion and commentary, case reports and extended reports. The manuscript management system is completely online and includes a very quick and fair peerreview system, which is all easy to use. Visit http://www.dovepress.com/ testimonials.php to read real quotes from published authors.

Submit your manuscript here: http://www.dovepress.com/international-journal-of-nephrology-and-renovascular-disease-journal 